\title{
INITIAL VALUE PROBLEMS FOR FUNCTIONAL AND NEUTRAL FUNCTIONAL HADAMARD TYPE FRACTIONAL DIFFERENTIAL INCLUSIONS
}

\author{
BASHIR AHMAD AND SOTIRIS K. NTOUYAS
}

Received 18 April, 2015

\begin{abstract}
This paper is concerned with the existence of solutions for initial value problems of functional and neutral functional Hadamard type fractional differential inclusions. We apply appropriate fixed point theorems for multivalued maps to obtain the existence results for the given problems covering convex as well as non-convex cases. Some illustrative examples are also presented.
\end{abstract}

2010 Mathematics Subject Classification: 34A60; 34A08

Keywords: fractional differential inclusions, neutral fractional differential inclusions, existence, fixed point

\section{INTRODUCTION}

In this paper we study the existence of solutions for initial value problems of functional and neutral functional Hadamard type fractional differential inclusions given by

$$
\begin{gathered}
D^{\alpha} y(t) \in F\left(t, y_{t}\right), \text { for each } t \in J:=[1, b], \quad 0<\alpha<1, \\
y(t)=\vartheta(t), \quad t \in[1-r, 1],
\end{gathered}
$$

and

$$
\begin{gathered}
D^{\alpha}\left[y(t)-g\left(t, y_{t}\right)\right] \in F\left(t, y_{t}\right), t \in J, \\
y(t)=\vartheta(t), t \in[1-r, 1],
\end{gathered}
$$

where $D^{\alpha}$ is the Hadamard fractional derivative, $F: J \times C([-r, 0], \mathbb{R}) \rightarrow \mathcal{P}(\mathbb{R})$ $(\mathcal{P}(\mathbb{R})$ is the family of all nonempty subjects of $\mathbb{R})$ is a given function and $\vartheta \in C([1-$ $r, 1], \mathbb{R})$ with $\vartheta(1)=0$ and $g: J \times C([-r, 0], \mathbb{R}) \rightarrow \mathbb{R}$ is a given function such that $g(1, \vartheta)=0$. For any function $y$ defined on $[1-r, b]$ and any $t \in J$, let $y_{t}$ denote the element of $C([-r, 0], \mathbb{R})$ defined by $y_{t}(\chi)=y(t+\chi), \quad \chi \in[-r, 0]$. Here $y_{t}(\cdot)$ represents the history of the state from time $t-r$ up to the present time $t$.

Functional and neutral functional differential equations arise in the mathematical modelling of biological, physical, and engineering problemss, see, for example, the texts [19], [17] and the references cited therein. 
Fractional order operators are found to be valuable tools in describing several phenomena occurring in applied sciences and engineering. Examples include viscoelasticity, electrochemistry, control, porous media, electromagnetic, etc. (see [18,21-23]).

Fractional differential equations involving Riemann-Liouville and Caputo type fractional derivatives have extensively been studied by several researchers $[1-4,6$, $7,15,24,25]$. On the other hand, Hadamard type fractional differential equations are not studied in depth and many aspects of these equations need further attention. It is imperative to note that the definition of Hadamard fractional derivative contains a logarithmic function of arbitrary exponent in contrast to aforementioned derivatives. For details, we refer the reader to the papers [9-11] and references cited therein.

The IVPs (1.1)-(1.2) and (1.3)-(1.4) involving Riemann-Liouville fractional derivative and infinite delay were studied in [8] while the case of functional fractional Hadamard type differential equations has recently been discussed in [5]. Here we extend the problems addressed in [5] to the multi-valued case.

The purpose of this paper is to establish some existence results for the problems (1.1)-(1.2) and (1.3)-(1.4) for convex and non-convex values of the multivalued maps involved in the problems. Our main results rely on the nonlinear alternative of LeraySchauder type and a fixed point theorem for contraction multivalued maps due to Covitz and Nadler.

The paper is organized as follows. Section 2 contains some preliminaries needed for the sequel. In Section 3, we establish the existence results for the problem (1.1)(1.2) while the results for the problem (1.3)-(1.4) are presented in Section 4. The paper concludes with illustrating examples.

\section{PRELIMINARIES}

Here we fix notations and state some definitions, and preliminary facts that we need in the sequel.

The space $C(J, \mathbb{R})$ denote the Banach space of all continuous functions from $J$ into $\mathbb{R}$ equipped with the norm $\|y\|_{\infty}:=\sup \{|y(t)|: t \in J\}$ and $C([-r, 0], \mathbb{R})$ is endowed with norm $\|\cdot\|_{C}$ defined by $\|\vartheta\|_{C}:=\sup \{|\vartheta(\chi)|:-r \leq \chi \leq 0\}$.

Definition 1 ([18]). The Hadamard derivative of fractional order $q$ for a function $g:[1, \infty) \rightarrow \mathbb{R}$ is defined as

$D^{q} g(t)=\frac{1}{\Gamma(n-q)}\left(t \frac{d}{d t}\right)^{n} \int_{1}^{t}\left(\log \frac{t}{s}\right)^{n-q-1} \frac{g(s)}{s} d s, n-1<q<n, n=[q]+1$, where $[q]$ denotes the integer part of the real number $q$ and $\log (\cdot)=\log _{e}(\cdot)$.

Definition 2 ([18]). The Hadamard fractional integral of order $q$ for a function $g$ is defined as

$$
I^{q} g(t)=\frac{1}{\Gamma(q)} \int_{1}^{t}\left(\log \frac{t}{s}\right)^{q-1} \frac{g(s)}{s} d s, q>0,
$$

provided the integral exists. 
For the convenience of the reader, we briefly describe some basic definitions.

Let $(\mathscr{D},\|\cdot\|)$ denote a normed space and $\mathcal{P}_{1}=\mathscr{P}(\mathscr{D}):=\{A \subset \mathscr{D}: A \neq \varnothing\}$. Then we define $\mathcal{P}_{c l}(\mathscr{D})=\left\{\mathscr{D}_{1} \in \mathcal{P}_{1}: \mathscr{D}_{1}\right.$ is closed $\}, \mathscr{P}_{b}(\mathscr{D})=\left\{\mathscr{D}_{1} \in \mathscr{P}_{1}: \mathscr{D}_{1}\right.$ is bounded $\}$, $\mathcal{P}_{c p}(\mathscr{D})=\left\{\mathscr{D}_{1} \in \mathscr{P}_{1}: \mathscr{D}_{1}\right.$ is compact $\}$, and $\mathcal{P}_{c p, c}(\mathscr{D})=\left\{\mathscr{D}_{1} \in \mathcal{P}_{1}: \mathscr{D}_{1}\right.$ is compact and convex $\}$.

Definition 3. A multi-valued map $\mathscr{E}: \mathscr{D} \rightarrow \mathcal{P}_{1}$ is

(a) convex (closed) valued if $\mathscr{E}(a)$ is convex (closed) for all $a \in \mathscr{D}$;

(b) bounded on bounded sets if $\mathcal{G}(\mathbb{B})=\cup_{x \in \mathbb{B}} \mathcal{E}(x)$ is bounded in $\mathscr{D}$ for all $\mathbb{B} \in$ $\mathscr{P}_{b}(\mathscr{D})\left(\right.$ i.e. $\sup _{x \in \mathbf{B}}\{\sup \{|y|: y \in G(x)\}\}<\infty$ );

(c) upper semi-continuous (u.s.c.) on $\mathscr{D}$ if for each $a_{0} \in \mathscr{D}$, the set $\mathcal{E}\left(a_{0}\right)$ is a nonempty closed subset of $\mathscr{D}$, and if for each open set $\mathcal{N}$ of $\mathscr{D}$ containing $\mathscr{E}\left(a_{0}\right)$, there exists an open neighborhood $\mathcal{N}_{0}$ of $a_{0}$ such that $\mathscr{G}\left(\mathcal{N}_{0}\right) \subseteq \mathcal{N}$;

(d) completely continuous if $\mathscr{E}(\mathbb{B})$ is relatively compact for every $\mathbb{B} \in \mathcal{P}_{b}(\mathscr{D})$.

Definition 4. The map $\mathcal{E}$ has a fixed point if there is $a \in \mathscr{D}$ such that $a \in \mathscr{E}(a)$. The fixed point set of the multivalued operator $\mathcal{E}$ will be denoted by Fix $\mathscr{G}$.

Definition 5. The map $\mathscr{E}: J \rightarrow \mathcal{P}_{c l}(\mathbb{R})$ is said to be measurable if for every $b \in \mathbb{R}$, the function $t \longmapsto d(b, \mathcal{E}(t))=\inf \{|b-c|: c \in \mathcal{E}(t)\}$ is measurable.

Definition 6. A multivalued map $F: J \times \mathbb{R} \rightarrow \mathcal{P}(\mathbb{R})$ is said to be Carathéodory if

(i) $t \longmapsto F(t, y)$ is measurable for each $x \in \mathbb{R}$;

(ii) $y \longmapsto F(t, y)$ is upper semicontinuous for almost all $t \in J$.

Further a Carathéodory function $F$ is called $L^{1}$-Carathéodory if

(i i i ) for each $\rho>0$, there exists $\varphi_{\rho} \in L^{1}\left(J, \mathbb{R}^{+}\right)$such that

$$
\|F(t, y)\|=\sup \{|v|: v \in F(t, y)\} \leq \varphi_{\rho}(t)
$$

for all $\|y\| \leq \rho$ and for a.e. $t \in J$.

\section{EXISTENCE RESUlTS FOR THE PROBLEM (1.1)-(1.2)}

In this section, we establish the existence criteria for the problem (1.1)-(1.2). Before presenting the main results, we outline some auxiliary material.

For each $y \in C(J, \mathbb{R})$, define the set of selections of $F$ by

$$
S_{F, y}:=\left\{v \in L^{1}(J, \mathbb{R}): v(t) \in F\left(t, y_{t}\right) \text { for a.e. } t \in J\right\} .
$$

Definition 7. A function $x \in C^{1}([1-r, b], \mathbb{R})$ is called a solution of problem (1.1)(1.2) if there exists a function $v \in L^{1}(J, \mathbb{R})$ with $v(t) \in F\left(t, y_{t}\right)$, a.e. $J$ such that $D^{\alpha} y(t)=v(t)$ for a.e. $t \in J$ and $y(t)=\vartheta(t), t \in[1-r, 1]$. 
To discuss the existence of the solutions for the initial value problem (1.1)-(1.2) when the right-hand side is convex valued (upper semicontinuous case), we need the following lemmas.

Lemma 1 ([16]). (Nonlinear alternative for Kakutani maps.) Let $E$ be a Banach space, $C$ a closed convex subset of $E, U$ an open subset of $C$ and $0 \in U$. Suppose that $F: \bar{U} \rightarrow \mathcal{P}_{c p, c}(C)$ is a upper semicontinuous compact map. Then either

(i) $F$ has a fixed point in $\bar{U}$, or

(ii) there is $a u \in \partial U$ and $\lambda \in(0,1)$ with $u \in \lambda F(u)$.

Lemma 2 ([20]). Let $X$ be a Banach space. Let $F: J \times X \rightarrow \mathcal{P}_{c p, c}(X)$ be an $L^{1}$ - Carathéodory multivalued map and let $\Theta$ be a linear continuous mapping from $L^{1}(J, X)$ to $C(J, X)$. Then the operator

$$
\Theta \circ S_{F}: C(J, X) \rightarrow \mathcal{P}_{c p, c}(C(J, X)), x \mapsto\left(\Theta \circ S_{F}\right)(x)=\Theta\left(S_{F, x}\right)
$$

is a closed graph operator in $C(J, X) \times C(J, X)$.

Theorem 1. Assume that

$\left(H_{1}\right) \quad F: J \times \mathbb{R} \rightarrow \mathcal{P}(\mathbb{R})$ is $L^{1}$-Carathéodory and has nonempty compact and convex values;

$\left(\mathrm{H}_{2}\right)$ there exists a continuous nondecreasing function $\beta:[0, \infty) \rightarrow(0, \infty)$ and $a$ function $\zeta \in C\left(J, \mathbb{R}^{+}\right)$such that

$$
\|F(t, y)\|_{\mathcal{P}}:=\sup \{|v|: v \in F(t, y)\} \leq \zeta(t) \beta\left(\|y\|_{C}\right),
$$

for each $(t, y) \in J \times C([-r, 0], \mathbb{R})$;

$\left(\mathrm{H}_{3}\right)$ there exists a constant $\sigma>0$ such that

$$
\frac{\sigma}{\beta(\sigma)\|\zeta\|_{\infty} \frac{(\log b)^{\alpha}}{\Gamma(\alpha+1)}}>1 .
$$

Then the initial value problem (1.1)-(1.2) has at least one solution on $[1-r, b]$.

Proof. Define an operator $\Omega_{F}: C([1-r, b], \mathbb{R}) \rightarrow \mathcal{P}(C([1-r, b], \mathbb{R}))$ by

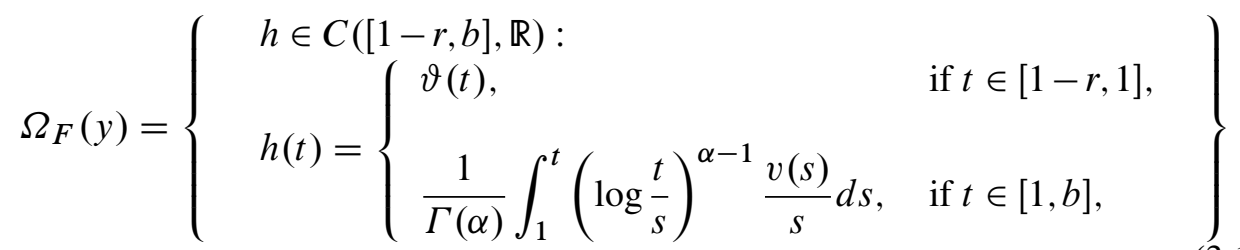

for $v \in S_{F, y}$. It will be shown that the operator $\Omega_{F}$ satisfies the assumptions of Lemma 1. Firstly we observe that $\Omega_{F}$ is convex for each $x \in C([1-r, b], \mathbb{R})$ since $S_{F, x}$ is convex ( $F$ has convex values). Next, we show that $\Omega_{F}$ maps bounded sets into bounded sets in $C([1-r, b], \mathbb{R})$. For a positive number $r$, let $B_{r}=\{y \in C([1-$ 
$\left.r, b], \mathbb{R}):\|y\|_{[1-r, b]} \leq r\right\}$ be a bounded ball in $C([1-r, b], \mathbb{R})$. Then, for each $h \in$ $\Omega_{F}(y), y \in B_{r}$, there exists $v \in S_{F, y}$ such that

$$
h(t)=\frac{1}{\Gamma(\alpha)} \int_{1}^{t}\left(\log \frac{t}{s}\right)^{\alpha-1} \frac{v(s)}{s} d s .
$$

Then for $t \in J$, we have

$$
\begin{aligned}
|h(t)| & \leq \frac{1}{\Gamma(\alpha)} \int_{1}^{t}\left(\log \frac{t}{s}\right)^{\alpha-1}|v(s)| \frac{d s}{s} \\
& \leq \frac{\beta\left(\|y\|_{[1-r, b]}\right)\|\zeta\|_{\infty}}{\Gamma(\alpha)} \int_{1}^{t}\left(\log \frac{t}{s}\right)^{\alpha-1} \frac{d s}{s} \\
& \leq \frac{\beta\left(\|y\|_{[1-r, b]}\right)\|\zeta\|_{\infty}}{\Gamma(\alpha+1)}(\log b)^{\alpha} .
\end{aligned}
$$

Thus

$$
\|h\| \leq \frac{\beta(r)\|\zeta\|_{\infty}}{\Gamma(\alpha+1)}(\log b)^{\alpha}:=\tilde{\ell} .
$$

Now we show that $\Omega_{F}$ maps bounded sets into equicontinuous sets of $C([1-r, b], \mathbb{R})$. Let $t_{1}, t_{2} \in J$ with $t_{1}<t_{2}$ and $x \in B_{r}$. For each $h \in \Omega_{F}(y)$, we obtain

$$
\begin{aligned}
\left|h\left(t_{2}\right)-h\left(t_{1}\right)\right| & \leq \frac{1}{\Gamma(\alpha)} \mid \int_{1}^{t_{1}}\left[\left(\log \frac{t_{2}}{s}\right)^{\alpha-1}-\left(\log \frac{t_{1}}{s}\right)^{\alpha-1}\right] f\left(s, y_{s}\right) \frac{d s}{s} \\
& +\frac{1}{\Gamma(\alpha)} \int_{t_{1}}^{t_{2}}\left(\log \frac{t_{2}}{s}\right)^{\alpha-1} f\left(s, y_{s}\right) \frac{d s}{s} \mid \\
& \leq \frac{\beta(r)\|\zeta\|_{\infty}}{\Gamma(\alpha)} \int_{1}^{t_{1}}\left[\left(\log \frac{t_{2}}{s}\right)^{\alpha-1}-\left(\log \frac{t_{1}}{s}\right)^{\alpha-1}\right] \frac{d s}{s} \\
& +\frac{\beta(r)\|\zeta\|_{\infty}}{\Gamma(\alpha)} \int_{t_{1}}^{t_{2}}\left(\log \frac{t_{2}}{s}\right)^{\alpha-1} \frac{d s}{s} .
\end{aligned}
$$

Clearly the right hand side of the above inequality tends to zero independent of $x \in$ $B_{r}$ as $t_{2}-t_{1} \rightarrow 0$. As $\Omega_{F}$ satisfies the above three assumptions, it follows by the Ascoli-Arzelá theorem that $\Omega_{F}: C([1-r, b], \mathbb{R}) \rightarrow \mathcal{P}(C([1-r, b], \mathbb{R}))$ is completely continuous.

In our next step, we show that $\Omega_{F}$ is upper semicontinuous. It is known [14, Proposition 1.2] that $\Omega_{F}$ will be upper semicontinuous if we establish that it has a closed graph, since $\Omega_{F}$ is already shown to be completely continuous. Thus we will prove that $\Omega_{F}$ has a closed graph. Let $y_{n} \rightarrow y_{*}, h_{n} \in \Omega_{F}\left(y_{n}\right)$ and $h_{n} \rightarrow h_{*}$. Then we need to show that $h_{*} \in \Omega_{F}\left(y_{*}\right)$. Associated with $h_{n} \in \Omega_{F}\left(y_{n}\right)$, there exists 
$v_{n} \in S_{F, y_{n}}$ such that for each $t \in J$,

$$
h_{n}(t)=\frac{1}{\Gamma(\alpha)} \int_{1}^{t}\left(\log \frac{t}{s}\right)^{\alpha-1} v_{n}(s) \frac{d s}{s} .
$$

Thus it suffices to show that there exists $v_{*} \in S_{F, y_{*}}$ such that for each $t \in J$,

$$
h_{*}(t)=\frac{1}{\Gamma(\alpha)} \int_{1}^{t}\left(\log \frac{t}{s}\right)^{\alpha-1} v_{*}(s) \frac{d s}{s} .
$$

Let us consider the linear operator $\Theta: L^{1}(J, \mathbb{R}) \rightarrow C(J, \mathbb{R})$ given by

$$
v \mapsto \Theta(v)(t)=\frac{1}{\Gamma(\alpha)} \int_{1}^{t}\left(\log \frac{t}{s}\right)^{\alpha-1} v(s) \frac{d s}{s} .
$$

Notice that

$$
\left\|h_{n}(t)-h_{*}(t)\right\|=\left\|\frac{1}{\Gamma(\alpha)} \int_{1}^{t}\left(\log \frac{t}{s}\right)^{\alpha-1}\left(v_{n}(s)-v_{*}(s)\right) \frac{d s}{s}\right\| \rightarrow 0, \text { as } n \rightarrow \infty .
$$

Thus, it follows by Lemma 2 that $\Theta \circ S_{F}$ is a closed graph operator. Further, we have $h_{n}(t) \in \Theta\left(S_{F, y_{n}}\right)$. Since $y_{n} \rightarrow y_{*}$, therefore, we have

$$
h_{*}(t)=\frac{1}{\Gamma(\alpha)} \int_{1}^{t}\left(\log \frac{t}{s}\right)^{\alpha-1} v_{*}(s) \frac{d s}{s},
$$

for some $v_{*} \in S_{F, y_{*}}$.

Finally, we show there exists an open set $U \subseteq C(J, \mathbb{R})$ with $y \notin \Omega_{F}(y)$ for any $\lambda \in$ $(0,1)$ and all $y \in \partial U$. Let $\lambda \in(0,1)$ and $y \in \lambda \Omega_{F}(y)$. Then there exists $v \in L^{1}(J, \mathbb{R})$ with $v \in S_{F, y}$ such that, for $t \in J$, we have

$$
y(t)=\lambda\left(\frac{1}{\Gamma(\alpha)} \int_{1}^{t}\left(\log \frac{t}{s}\right)^{\alpha-1} v(s) \frac{d s}{s}\right) .
$$

By the assumption $\left(H_{2}\right)$, for each $t \in J$, we get

$$
\begin{aligned}
|y(t)| & \leq \frac{1}{\Gamma(\alpha)} \int_{1}^{t}\left(\log \frac{t}{s}\right)^{\alpha-1} \zeta(s) \beta\left(\left\|y_{s}\right\|\right) \frac{d s}{s} \\
& \leq \frac{\|\zeta\|_{\infty} \beta\left(\|y\|_{[1-r, b]}\right)}{\Gamma(\alpha+1)}(\log b)^{\alpha},
\end{aligned}
$$

which can be expressed as

$$
\frac{\|y\|_{[1-r, b]}}{\beta\left(\|y\|_{[1-r, b]}\right)\|\zeta\|_{\infty} \frac{(\log b)^{\alpha}}{\Gamma(\alpha+1)}} \leq 1 .
$$

In view of $\left(H_{3}\right)$, there exists $\sigma$ such that $\|y\|_{[1-r, b]} \neq \sigma$. Let us set

$$
U=\left\{y \in C([1-r, b], \mathbb{R}):\|y\|_{[1-r, b]}<\sigma\right\} .
$$


Note that the operator $\Omega_{F}: \bar{U} \rightarrow \mathcal{P C}([1-r, b], \mathbb{R})$ is upper semicontinuous and completely continuous. From the choice of $U$, there is no $y \in \partial U$ such that $y \in$ $\lambda \Omega_{F}(y)$ for some $\lambda \in(0,1)$. Consequently, by the nonlinear alternative of LeraySchauder type (Lemma 1), we deduce that $\Omega_{F}$ has a fixed point $y \in \bar{U}$ which is a solution of the problem (1.1)-(1.2). This completes the proof.

Next we prove the existence of solutions for the problem (1.1)-(1.2) with a nonconvex valued right hand side (Lipschitz case) by applying a fixed point theorem for multivalued map due to Covitz and Nadler. Let us first introduce terminology.

Let $(X, d)$ be a metric space induced from the normed space $(X ;\|\cdot\|)$. Consider $H_{d}: \mathcal{P}(X) \times \mathcal{P}(X) \rightarrow \mathbb{R} \cup\{\infty\}$ given by

$$
H_{d}(A, B)=\max \left\{\sup _{a \in A} d(a, B), \sup _{b \in B} d(A, b)\right\},
$$

where $d(A, b)=\inf _{a \in A} d(a ; b)$ and $d(a, B)=\inf _{b \in B} d(a ; b)$.

Definition 8. A multivalued operator $N: X \rightarrow \mathcal{P}_{c l}(X)$ is called:

(a) $\gamma$-Lipschitz if and only if there exists $\gamma>0$ such that

$$
H_{d}(N(x), N(y)) \leq \gamma d(x, y) \text { for each } x, y \in X ;
$$

(b) a contraction if and only if it is $\gamma$-Lipschitz with $\gamma<1$.

Lemma 3 ([13]). Let $(X, d)$ be a complete metric space. If $N: X \rightarrow \mathcal{P}_{c l}(X)$ is a contraction, then FixN $\neq \varnothing$.

Theorem 2. Assume that:

$\left(H_{4}\right) F: J \times \mathbb{R} \rightarrow \mathcal{P}_{c p}(\mathbb{R})$ is such that $F(\cdot, y): J \rightarrow \mathcal{P}_{c p}(\mathbb{R})$ is measurable for each $y \in \mathbb{R}$.

$\left(H_{5}\right) H_{d}(F(t, y), F(t, \bar{y})) \leq \ell(t)\|y-\bar{y}\|_{C}$ for almost all $t \in J$ and $y, \bar{y}$ $\in C([-r, 0], \mathbb{R})$ with $\ell \in C\left(J, \mathbb{R}^{+}\right)$and $d(0, F(t, 0)) \leq \ell(t)$ for almost all $t \in J$.

Then, if $\frac{(\log b)^{\alpha}}{\Gamma(\alpha+1)}\|\ell\|_{\infty}<1$, the initial value problem (1.1)-(1.2) has at least one solution on $[1-r, b]$.

Proof. Observe that the set $S_{F, y}$ is nonempty for each $x \in C([1-r, b], \mathbb{R})$ by the assumption $\left(H_{4}\right)$, so $F$ has a measurable selection (see Theorem III.6 [12]). Now we show that the operator $\Omega_{F}$, defined by (3.1), satisfies the hypothesis of Lemma 3. To show that $\Omega_{F}(y) \in \mathcal{P}_{c l}(C([1-r, b], \mathbb{R}))$ for each $y \in C([1-r, b], \mathbb{R})$, let $\left\{u_{n}\right\}_{n \geq 0} \in$ $\Omega_{F}(y)$ be such that $u_{n} \rightarrow u(n \rightarrow \infty)$ in $C([1-r, b], \mathbb{R})$. Then $u \in C([1-r, b], \mathbb{R})$ and there exists $v_{n} \in S_{F, y_{n}}$ such that, for each $t \in J$,

$$
u_{n}(t)=\frac{1}{\Gamma(\alpha)} \int_{1}^{t}\left(\log \frac{t}{s}\right)^{\alpha-1} v_{n}(s) \frac{d s}{s} .
$$


As $F$ has compact values, we pass onto a subsequence (if necessary) to obtain that $v_{n}$ converges to $v$ in $L^{1}(J, \mathbb{R})$. Thus, $v \in S_{F, y}$ and for each $t \in J$, we have

$$
u_{n}(t) \rightarrow u(t)=\frac{1}{\Gamma(\alpha)} \int_{1}^{t}\left(\log \frac{t}{s}\right)^{\alpha-1} v(s) \frac{d s}{s} .
$$

Hence, $u \in \Omega(y)$.

Next we show that there exists $\delta<1\left(\delta:=\frac{(\log b)^{\alpha}}{\Gamma(\alpha+1)}\|\ell\|_{\infty}\right)$ such that

$$
H_{d}\left(\Omega_{F}(y), \Omega_{F}(\bar{y})\right) \leq \delta\|y-\bar{y}\|_{C} \text { for each } y, \bar{y} \in C([1-r, b], \mathbb{R}) .
$$

Let $y, \bar{y} \in C([1-r, b], \mathbb{R})$ and $h_{1} \in \Omega_{F}(y)$. Then there exists $v_{1}(t) \in F\left(t, y_{t}\right)$ such that, for each $t \in J$,

$$
h_{1}(t)=\frac{1}{\Gamma(\alpha)} \int_{1}^{t}\left(\log \frac{t}{s}\right)^{\alpha-1} v_{1}(s) \frac{d s}{s} .
$$

By $\left(H_{5}\right)$, we have

$$
H_{d}(F(t, y), F(t, \bar{y})) \leq \ell(t)\|y-\bar{y}\|_{C} .
$$

So, there exists $w \in F\left(t, \bar{y}_{t}\right)$ such that

$$
\left|v_{1}(t)-w\right| \leq \ell(t)\|y-\bar{y}\|_{C}, \quad t \in J .
$$

Define $U: J \rightarrow \mathcal{P}(\mathbb{R})$ by

$$
U(t)=\left\{w \in \mathbb{R}:\left|v_{1}(t)-w\right| \leq \ell(t)\|y-\bar{y}\|_{C}\right\} .
$$

Since the multivalued operator $U(t) \cap F\left(t, \bar{y}_{t}\right)$ is measurable (Proposition III.4 [12]), there exists a function $v_{2}(t)$ which is a measurable selection for $U$. So $v_{2}(t) \in$ $F\left(t, \bar{y}_{t}\right)$ and for each $t \in J$, we have $\left|v_{1}(t)-v_{2}(t)\right| \leq \ell(t)\|y-\bar{y}\|_{C}$.

For each $t \in J$, let us define

$$
h_{2}(t)=\frac{1}{\Gamma(\alpha)} \int_{1}^{t}\left(\log \frac{t}{s}\right)^{\alpha-1} v_{2}(s) \frac{d s}{s} .
$$

Thus,

$$
\begin{aligned}
\left|h_{1}(t)-h_{2}(t)\right| & \leq \frac{1}{\Gamma(\alpha)} \int_{1}^{t}\left(\log \frac{t}{s}\right)^{\alpha-1}\left|v_{1}(s)-v_{2}(s)\right| \frac{d s}{s} \\
& \leq \frac{1}{\Gamma(\alpha)} \int_{1}^{t}\left(\log \frac{t}{s}\right)^{\alpha-1} \ell(s)\|y-\bar{y}\|_{C} \frac{d s}{s} \\
& \leq \frac{1}{\Gamma(\alpha)} \int_{1}^{b}\left(\log \frac{t}{s}\right)^{\alpha-1} \ell(s)\|y-\bar{y}\|_{[1-r, b]} \frac{d s}{s} \\
& \leq \frac{(\log b)^{\alpha}}{\Gamma(\alpha+1)}\|\ell\|_{\infty}\|y-\bar{y}\|_{[1-r, b]} .
\end{aligned}
$$


Hence,

$$
\left\|h_{1}-h_{2}\right\| \leq \frac{(\log b)^{\alpha}}{\Gamma(\alpha+1)}\|\ell\|_{\infty}\|y-\bar{y}\|_{[1-r, b]} .
$$

Analogously, interchanging the roles of $y$ and $\bar{y}$, we obtain

$$
H_{d}\left(\Omega_{F}(y), \Omega_{F}(\bar{y})\right) \leq \frac{1}{\Gamma(\alpha+1)}\|\ell\|_{\infty}\|y-\bar{y}\|_{[1-r, b]} .
$$

Since $\Omega_{F}$ is a contraction, it follows by Lemma 3 that $\Omega_{F}$ has a fixed point $x$ which is a solution of (1.1)-(1.2). This completes the proof.

\section{EXISTENCE RESUlTS FOR THE PROBLEM (1.3)-(1.4)}

This section is devoted to some results dealing with the existence of solutions for the problem (1.3)-(1.4).

Definition 9. A function $y \in C^{1}([1-r, b], \mathbb{R})$, is said to be a solution of (1.3)(1.4) if there exists a function $v \in L^{1}([1, b], \mathbb{R})$ with $v(t) \in F\left(t, y_{t}\right)$, a.e. [1,b] such that $D^{\alpha}\left[y(t)-g\left(t, y_{t}\right)\right]=v(t)$ on $J$, and the condition $y(t)=\vartheta(t)$ on $[1-r, 1]$.

Theorem 3. Assume that $\left(H_{1}\right)-\left(H_{2}\right)$ hold. Further we suppose that

$\left(H_{6}\right)$ the function $g$ is continuous and completely continuous, and for any bounded set $B$ in $C([1-r, b], \mathbb{R})$, the set $\left\{t \rightarrow g\left(t, y_{t}\right): y \in B\right\}$ is equicontinuous in $C([1, b], \mathbb{R})$, and there exist constants $0 \leq d_{1}<1, d_{2} \geq 0$ such that

$$
|g(t, u)| \leq d_{1}\|u\|_{C}+d_{2}, \quad t \in[1, b], u \in C([-r, 0], \mathbb{R}) .
$$

$\left(\mathrm{H}_{7}\right)$ there exists a constant $M>0$ such that

$$
\frac{\left(1-d_{1}\right) M}{d_{2}+\frac{\|\zeta\|_{\infty} \beta(M)}{\Gamma(\alpha+1)}(\log b)^{\alpha}}>1
$$

Then the IVP (1.3)-(1.4) has at least one solution on $[1-r, b]$.

Proof. Define the operator $N: C([1-r, b], \mathbb{R}) \rightarrow \mathcal{P} C([1-r, b], \mathbb{R})$ by

$$
N(y)= \begin{cases}h \in C([1-r, b], \mathbb{R}): & \text { if } t \in[1-r, 1], \\
h(t)=\left\{\begin{array}{ll}
\vartheta(t), & \text { if } t \in[1, b], \\
g\left(t, x_{t}\right)+\frac{1}{\Gamma(\alpha)} \int_{1}^{t}\left(\log \frac{t}{s}\right)^{\alpha-1} \frac{v(s)}{s} d s,
\end{array}\right\}\end{cases}
$$

for $v \in S_{F, y}$. 
Using $\left(H_{6}\right)$, it suffices to show that the operator $N_{1}: C([1-r, b], \mathbb{R}) \rightarrow C([1-$ $r, b], \mathbb{R})$ defined by

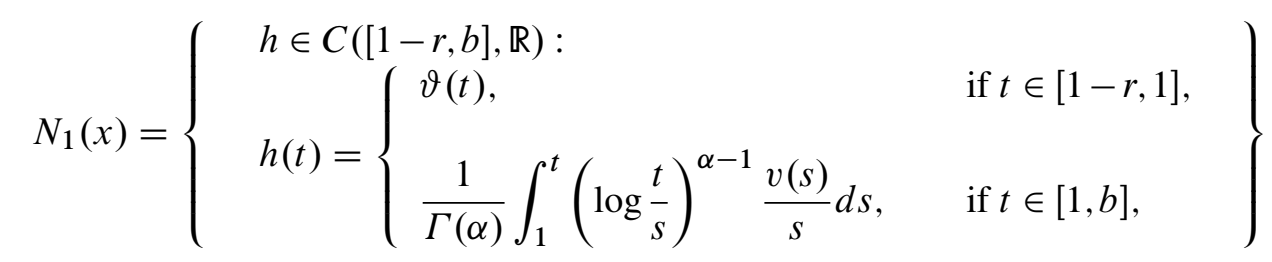

for $v \in S_{F, y}$, is continuous and completely continuous. The proof is similar to that of Theorem 1. So we omit the details.

Next we now show that there exists an open set $U \subseteq C([1-r, b], \mathbb{R})$ with $y \neq$ $\lambda N(y)$ for $\lambda \in(0,1)$ and $y \in \partial U$.

Let $y \in C([1-r, b], \mathbb{R})$ be such that $y=\lambda N(y)$ for some $0<\lambda<1$. Thus, for each $t \in[1, b]$, we have

$$
y(t)=\lambda\left(g\left(t, y_{t}\right)+\frac{1}{\Gamma(\alpha)} \int_{1}^{t}\left(\log \frac{t}{s}\right)^{\alpha-1} \frac{v(s)}{s} d s\right) .
$$

For each $t \in J$, it follows by $\left(H_{2}\right)$ and $\left(H_{4}\right)$ that

$$
\begin{aligned}
|y(t)| & \leq d_{1}\left\|y_{t}\right\|_{C}+d_{2}+\frac{1}{\Gamma(\alpha)} \int_{1}^{t}\left(\log \frac{t}{s}\right)^{\alpha-1} \zeta(s) \beta\left(\left\|y_{s}\right\|_{C}\right) \frac{d s}{s} \\
& \leq d_{1}\left\|y_{t}\right\|_{C}+d_{2}+\frac{\|\zeta\|_{\infty} \beta\left(\|y\|_{[1-r, b]}\right)}{\Gamma(\alpha+1)}(\log b)^{\alpha}
\end{aligned}
$$

which yields

$$
\left(1-d_{1}\right)\|y\|_{[1-r, b]} \leq d_{2}+\frac{\|\zeta\|_{\infty} \beta\left(\|y\|_{[1-r, b]}\right)}{\Gamma(\alpha+1)}(\log b)^{\alpha} .
$$

In consequence, we get

$$
\frac{\left(1-d_{1}\right)\|y\|_{[1-r, b]}}{d_{2}+\frac{\|\zeta\|_{\infty} \beta\left(\|y\|_{[1-r, b]}\right)}{\Gamma(\alpha+1)}(\log b)^{\alpha}} \leq 1 .
$$

In view of $\left(H_{7}\right)$, there exists $M$ such that $\|y\|_{[1-r, b]} \neq M$. Let us set

$$
U=\left\{y \in C([1-r, b], \mathbb{R}):\|y\|_{[1-r, b]}<M\right\} .
$$

Note that the operator $N_{1}: \bar{U} \rightarrow C([1-r, b], \mathbb{R})$ is continuous and completely continuous. From the choice of $U$, there is no $y \in \partial U$ such that $y=\lambda N_{1} y$ for some $\lambda \in(0,1)$. Thus, by the nonlinear alternative of Leray-Schauder type (Lemma 1), we deduce that $N_{1}$ has a fixed point $y \in \bar{U}$ which is a solution of the problem (1.3)-(1.4). This completes the proof.

Theorem 4. Assume that $\left(H_{4}\right),\left(H_{5}\right)$ hold. In addition we suppose that: 
$\left(H_{8}\right)$ There exists a constant $L>0$ such that

$$
|g(t, x)-g(t, y)| \leq L\|x-y\|_{C}, \text { for all } t \in[1, b] \text { and } x, y \in C([-r, 0], \mathbb{R}) .
$$

Then, if $L+\frac{(\log b)^{\alpha}}{\Gamma(\alpha+1)}\|\ell\|_{\infty}<1$, the IVP (1.3)-(1.4) has at least one solution on $[1-r, b]$.

Proof. Since the proof is similar to that of Theorem 2, so it is omitted.

\section{EXAMPLES}

Example 1. For any function $\vartheta \in C([1-r, 1], \mathbb{R})$ with $\vartheta(1)=0$, consider the problem

$$
\begin{gathered}
D^{\alpha} y(t) \in F\left(t, y_{t}\right), \text { for each } t \in J:=[1, e], \quad 0<\alpha<1, \\
y(t)=\vartheta(t), \quad t \in[1-r, 1],
\end{gathered}
$$

where

$$
F\left(t, y_{t}\right)=\left[\frac{1}{4+e-t}\left(\frac{|y|_{t}}{2\left(1+|y|_{t}\right)}+\frac{1}{4}\right), \frac{1}{16}\left(1+e^{-t}\right)\right] .
$$

Clearly

$$
\left\|F\left(t, y_{t}\right)\right\|_{\mathcal{P}}:=\sup \left\{|u|: u \in F\left(t, y_{t}\right)\right\} \leq \frac{1}{4}\left(\frac{3}{4}\right), \quad y_{t} \in \mathbb{R} .
$$

With $\zeta(t)=1 / 4, \beta\left(\left\|y_{t}\right\|\right)=3 / 4$, by the condition $\left(H_{3}\right)$, we find that

$$
M>\frac{3}{16 \Gamma(\alpha+1)}, 0<\alpha<1 .
$$

Hence, by Theorem 1, the problem (5.1)-(5.2) has a solution on $[1-r, e]$.

Example 2. Let us consider the problem (5.1)-(5.2) with

$$
F\left(t, y_{t}\right)=\left[\frac{1}{16}, \frac{1}{\pi \sqrt{t+3}} \tan ^{-1}\left(y_{t}\right)+\frac{1}{12}\right] .
$$

Observe that

$$
H_{d}\left(F\left(t, y_{t}\right), F\left(t, \bar{y}_{t}\right)\right) \leq \frac{1}{\pi \sqrt{t+3}}\|y-\bar{y}\|_{C} .
$$

Letting $\ell(t)=\frac{1}{\pi \sqrt{t+3}}$, we find that $d(0, F(t, 0)) \leq \ell(t)$ for almost all $t \in J$ and $\frac{(\log b)^{\alpha}}{\Gamma(\alpha+1)}\|\ell\|_{\infty}=\frac{1}{2 \pi \Gamma(\alpha+1)}<1$, for $0<\alpha<1$. Thus all the conditions of Theorem 2 are satisfied. Hence, by the conclusion of Theorem 2, the problem (5.1)-(5.2) with (5.3) has a solution on $[1-r, e]$.

Remark 1. Illustrating examples for Theorems 3 and 4 can be constructed like Examples 1 and 2. 


\section{REFERENCES}

[1] R. Agarwal, Y. Zhou, and Y. He, "Existence of fractional neutral functional differential equations," Comput. Math. Appl., vol. 59, pp. 1095-1100, 2010, doi: 10.1016/j.camwa.2009.05.010.

[2] R. Agarwal, Y. Zhou, J. R. Wang, and X. Luo, "Fractional functional differential equations with causal operators in Banach spaces," Math. Comput. Modelling, vol. 54, pp. 1440-1452, 2014, doi: 10.1016/j.mcm.2011.04.016.

[3] B. Ahmad and S. Ntouyas, "Nonlinear fractional differential equations and inclusions of arbitrary order and multi-strip boundary conditions," Electron. J. Diff. Eqns, vol. 2012, no. 98, pp. 1-22, 2012.

[4] B. Ahmad and S. Ntouyas, "Integro-differential equations of fractional order with nonlocal fractional boundary conditions associated with financial asset model," Electron. J. Diff. Eqns, vol. 2013, no. 60, pp. 1-10, 2013.

[5] B. Ahmad and S. Ntouyas, "Initial value problems of fractional order hadamard-type functional differential equations," Electron. J. Diff. Equ., vol. 2015, no. 77, pp. 1-9, 2015.

[6] B. Ahmad, S. Ntouyas, and A. Alsaedi, "New existence results for nonlinear fractional differential equations with three-point integral boundary conditions," Adv. Differ. Equ., vol. 2011, no. 107384, pp. 1-11, 2011.

[7] B. Ahmad and S. Sivasundaram, "On four-point nonlocal boundary value problems of nonlinear integro-differential equations of fractional order," Appl. Math. Comput., vol. 217, pp. 480-487, 2010, doi: 10.1016/j.amc.2010.05.080.

[8] M. Benchohra, J. Henderson, S. K. Ntouyas, and A. Ouahab, "Existence results for fractional order functional differential equations with infinite delay," J. Math. Anal. Appl., vol. 338, pp. 1340-1350, 2008, doi: 10.1016/j.jmaa.2007.06.021.

[9] P. L. Butzer, A. A. Kilbas, and J. J. Trujillo, "Compositions of Hadamard-type fractional integration operators and the semigroup property," J. Math. Anal. Appl., vol. 269, pp. 387-400, 2002, doi: 10.1016/S0022-247X(02)00049-5.

[10] P. L. Butzer, A. A. Kilbas, and J. J. Trujillo, "Fractional calculus in the Mellin setting and Hadamard-type fractional integrals," J. Math. Anal. Appl., vol. 269, pp. 1-27, 2002, doi: 10.1016/S0022-247X(02)00001-X.

[11] P. L. Butzer, A. A. Kilbas, and J. J. Trujillo, "Mellin transform analysis and integration by parts for Hadamard-type fractional integrals," J. Math. Anal. Appl., vol. 270, pp. 1-15, 2002, doi: 10.1016/S0022-247X(02)00066-5.

[12] C. Castaing and M. Valadier, Convex Analysis and Measurable Multifunctions, ser. Lecture Notes in Mathematics. Berlin: Springer, 1977, vol. 580, doi: 10.1007/BFb0087685.

[13] H. Covitz and S. B. Nadler Jr, "Multivalued contraction mappings in generalized metric spaces," Israel J. Math., vol. 8, pp. 5-11, 1970, doi: 10.1007/BF02771543.

[14] K. Deimling, Multivalued Differential Equations, ser. Series in Nonlinear Analysis and Applications 1. Berlin-New York: Walter De Gruyter, 1992, vol. 580, doi: 10.1515/9783110874228.

[15] A. El-Sayed, "Nonlinear functional differential equations of arbitrary orders," Nonlin. Anal., vol. 33, pp. 181-186, 1998, doi: 10.1016/S0362-546X(97)00525-7.

[16] A. Granas and J. Dugundji, Fixed Point Theory. New York: Springer, 2003. doi: 10.1007/9780-387-21593-8.

[17] J. Hale and S. Verduyn Lunel, Introduction to Functional Differential Equations, ser. Applied Mathematical Sciences. New York: Springer, 1993, vol. 99, doi: 10.1007/978-1-4612-4342-7.

[18] A. Kilbas, H. Srivastava, and J. Trujillo, Theory and Applications of Fractional Differential Equations, ser. North-Holland Mathematics Studies. Amsterdam: Elsevier, 2006, vol. 204. 
[19] V. Kolmanovskii and A. Myshkis, Introduction to the Theory and Applications of FunctionalDifferential Equations, ser. Mathematics and its Applications. Dordrecht: Kluwer Academic Publishers, 1999, vol. 463, doi: 10.1007/978-94-017-1965-0.

[20] A. Lasota and Z. Opial, "An application of the Kakutani-Ky Fan theorem in the theory of ordinary differential equations,” Bull. Acad. Polon. Sci. Ser. Sci. Math. Astronom. Phys., vol. 13, pp. 781$786,1965$.

[21] K. Miller and B. Ross, An Introduction to the Fractional Calculus and Differential Equations. New York: John Wiley, 1993.

[22] I. Podlubny, Fractional Differential Equations, ser. Mathematics in Science and Engineering. San Diego: Academic Press, 1999, vol. 198.

[23] S. Samko, A. Kilbas, and O. Marichev, Fractional Integrals and Derivatives. Theory and Applications. Yverdon: Gordon and Breach, 1993.

[24] C. Yu and G. Gao, "Some results on a class of fractional functional differential equations," Commun. Appl. Nonlinear Anal., vol. 11, pp. 67-75, 2004.

[25] C. Yu and G. Gao, "Existence of fractional differential equations," J. Math. Anal. Appl., vol. 310, pp. 26-29, 2005, doi: 10.1016/j.jmaa.2004.12.015.

Authors' addresses

Bashir Ahmad

Nonlinear Analysis and Applied Mathematics (NAAM)-Research Group, Department of Mathematics, Faculty of Science, King Abdulaziz University, P.O. Box 80203, Jeddah 21589, Saudi Arabia

E-mail address: bashirahmad_qau@yahoo.com

Sotiris K. Ntouyas

Department of Mathematics, University of Ioannina, 45110 Ioannina, Greece; Nonlinear Analysis and Applied Mathematics (NAAM)-Research Group, Department of Mathematics, Faculty of Science, King Abdulaziz University, P.O. Box 80203, Jeddah 21589, Saudi Arabia

E-mail address: sntouyas@uoi.gr 